\title{
Overcoming the Temperature Effect on a Single Junction and Intermediate Band Solar Cells Using Optical Filter and SECs
}

S Vahedi

University of Tabriz

Mohammad Eskandari

University of Tabriz

Azeez Barzinjy

Salahaddin University - Erbil College of Education

Ali Rostami ( $D$ rostami@tabrizu.ac.ir)

University of Tabriz https://orcid.org/0000-0002-8727-4711

Mahboubeh Dolatyari

University of Tabriz

Ghasem Rostami

University of Tabriz

\section{Research Article}

Keywords: Efficiency, temperature, filter, single-junction solar cell, Intermediate Band Solar Cells (IBSCs), Detailed Balance model, ESCs

Posted Date: July 27th, 2021

DOl: https://doi.org/10.21203/rs.3.rs-673921/v1

License: (c) (i) This work is licensed under a Creative Commons Attribution 4.0 International License.

Read Full License 


\title{
Overcoming the Temperature effect on a single junction and Intermediate Band solar cells using Optical filter and SECs
}

\author{
S. Vahedi ${ }^{1}$, M. Eskandari ${ }^{1}$, Azeez A. Barzinjy ${ }^{2,3}$, A. Rostami ${ }^{1,4,5}$, M. Dolatyari ${ }^{5}$ and G. Rostami ${ }^{5}$ \\ ${ }^{1}$ OIC Research Group, University of Tabriz, Tabriz, 5166614761, Iran. \\ ${ }^{2}$ Department of Physics, College of Education, Salahaddin University-Erbil, Iraq \\ ${ }^{3}$ Physics Education Department, Education Faculty, Tishk International University, Erbil, Iraq \\ ${ }^{4}$ Photonics and Nanocrystal Research Lab. (PNRL), Faculty of Electrical and Computer Engineering, University of \\ Tabriz, Tabriz 5166614761, Iran. \\ ${ }^{5}$ SP- EPT Labs, ASEPE Company, Industrial Park of Advanced Technologies, 5364196795, Tabriz, Iran \\ rostami@tabrizu.ac.ir
}

\begin{abstract}
The performance of electronic devices, especially solar cells, at high temperatures is of primary interest to researchers. The design and construction of high-efficiency solar cells face some difficulties. One of these difficulties is the rising temperature, thus solar cells temperature assessment is essential to guarantee high performance. Normally, rising temperature, in solar cells, is associated with the normal ambient temperature and the produced internal temperature due to power dissipation. Accordingly, this investigation aims to reduce the effect of applied temperature from both sources. To reduce the destructive effect, the authors design a simple construction model that utilizes $\mathrm{SiO}_{2}$ and $\mathrm{Si}_{3} \mathrm{~N}_{4}$ as a filtering layer. The outcomes of this study show that the power conversion is optimum. $\mathrm{Si}$ and SiC Solar cells both with and without using a filter are evaluated and compared. Finally, enhancement in power conversion efficiency and other characteristics has been investigated. The intermediate band solar cells were evaluated with both internal and external temperature effects. To reduce the internal temperature, the authors utilized a novel method for extracting the hot carriers from different energy levels by using multilevel energy selective contacts (ESCs). It was shown that ESCs promote efficiency and break the Shockley-Queisser limit.
\end{abstract}

Keywords: Efficiency; temperature; filter; single-junction solar cell; Intermediate Band Solar Cells (IBSCs); Detailed Balance model; ESCs.

\section{Introduction}

Public awareness of the need to put an end to global warming and significant increases in oil prices has encouraged many countries around the world to adopt new energy policies that promote renewable energy applications to meet energy requirements and protect the environment. This is, the case, in both developed and developing countries. Solar energy can be mainly utilized in two ways, namely, either to use it directly for heating or cooling of air and water without using an intermediate electrical circuit or to convert it into electrical energy by using photovoltaic (PV) modules [1]. Direct conversion of solar radiation into electrical energy is the most convenient way of utilizing solar energy. The advantages of using the photovoltaic effect to generate electricity include no production of pollutants during operation, silence, long lifetime, and low maintenance. Moreover, solar energy is abundant, free, clean, and unlimited [1]. In general, a solar cell is an optoelectronic device that can directly convert solar energy into electrical 
energy [2]. The study of the impact of temperature (T) on solar cells is extremely important as, in terrestrial applications, they are generally exposed to a temperature ranging from $300 \mathrm{~K}$ to $450 \mathrm{~K}$ [1] and to even higher temperatures in space and concentrator systems. Temperature can affect how electricity flows through an electrical circuit by changing the speed at which the electrons travel. Also, since solar panels work best at certain weather and temperature conditions, engineers design ways to improve the efficiency of solar panels that operate in sub-optimal temperature conditions [2,3]. Designing and construction of solar cells which offer high efficiency have some difficulties that are addressed in this investigation. One of the most important problems is the high-temperature effect. Usually, the temperature is classified by the normal temperature of the environment and the temperature produced in the solar cell.

\section{Designing of filter}

As stated before, the impact of temperature can be investigated through the normal temperature of the environment and the temperature produced in the solar cell. Thus, in this study, the main purpose is to reduce the effect of applied temperature from both sides. To reduce the destructive effect, the authors design a simple construction model that uses $\mathrm{SiO}_{2}$ and $\mathrm{Si}_{3} \mathrm{~N}_{4}$ as a filtering layer. It shows that the power disturbing is extremely optimum. This study, also, presents a method for calculating the amount of reflected, transmitted spectrum by using the transfer matrix method in multilayered structures [4]. The transfer-matrix method is a method used in optics and acoustics to analyzes the propagation of electromagnetic or acoustic waves through a layered medium [4]. This is a relevant example of designing anti-reflective coatings and dielectric mirrors. Referring to Fig. 1 we will revisit the reflection and transmission of electromagnetic radiation through a thin film using the $2 \times 2$ matrix method.



Where $n_{0}, n_{1}$ and $n_{2}$ are the refractive indices respectively, $d_{1}$ and $d_{2}$ are the thickness of those layers. In addition, to consider the effect of temperature on the refractive indices, the following relation is used [5].

$$
n(T)=n\left(T_{0}\right)+\frac{\partial n}{\partial t}\left(T-T_{0}\right)
$$


In which $T$ is the temperature in media (junction in the solar cell), and $T_{0}$ equals $300 \mathrm{~K}$. The dielectric structure is described by the transfer-matrix method based on Maxwell's equations and there are simple continuity conditions for the electric field across boundaries from one medium to another. If the field is known at the beginning of a layer, the field at the end of the layer can be derived from a simple matrix operation. A stack of layers can then be represented as a system matrix, which is the product of the individual layer matrices. The final step of the method involves converting the system matrix back into reflection and transmission coefficients.

The reflection of light from a single interface between two media is described by the Fresnel equations. However, when there are multiple interfaces, such as in Fig. 1, the reflections themselves are also partially transmitted and then partially reflected. Depending on the exact path length, these reflections can interfere destructively or constructively. The overall reflection of a layer structure is the sum of an infinite number of reflections, which is cumbersome to calculate [4]. In this method, a boundary $2 \times 2$ matrix $D_{1}$ and diffusion $2 \times 2$ matrix $P_{1}$, defined for each layer as:

$$
\begin{aligned}
& D_{1}=\left[\begin{array}{cc}
1 & 1 \\
n_{1} \cos \theta_{1} & n_{2} \cos \theta_{2}
\end{array}\right] \\
& P_{1}=\left[\begin{array}{cc}
e^{-i \varphi} & 0 \\
0 & e^{i \varphi}
\end{array}\right]
\end{aligned}
$$

Where $n_{1}$ and $n_{2}$ are refractive indices, $\theta_{1}$ and $\theta_{2}$ are the angle of incident and reflected components and $\varphi$ is the changed phase of light as it propagates through the thin film given by:

$$
\varphi=\frac{2 \pi n d}{A} \cos \theta
$$

The resultant matrix is defined as the product of these characteristic matrices:

$$
\left(\begin{array}{ll}
M_{11} & M_{12} \\
M_{21} & M_{22}
\end{array}\right)=D_{0}^{-1}\left[\prod_{l=1}^{N} D_{l} P_{l} D_{l}^{-1}\right] D_{s}
$$

Where the elements of the matrix are written as:

$$
\begin{aligned}
& M_{11}=\frac{1}{2}\left(1+\frac{n_{3} \cos \theta_{3}}{n_{1} \cos \theta_{1}}\right) \cos \varphi+\frac{1}{2} i \sin \varphi\left(\frac{n_{2} \cos \theta_{2}}{n_{1} \cos \theta_{1}}-\frac{n_{3} \cos \theta_{3}}{n_{2} \cos \theta_{2}}\right) \\
& M_{12}=\frac{1}{2}\left(1-\frac{n_{3} \cos \theta_{3}}{n_{1} \cos \theta_{1}}\right) \cos \varphi+\frac{1}{2} i \sin \varphi\left(\frac{n_{2} \cos \theta_{2}}{n_{1} \cos \theta_{1}}-\frac{n_{3} \cos \theta_{3}}{n_{2} \cos \theta_{2}}\right)
\end{aligned}
$$




$$
\begin{aligned}
& M_{21}=\frac{1}{2}\left(1-\frac{n_{3} \cos \theta_{3}}{n_{1} \cos \theta_{1}}\right) \cos \varphi-\frac{1}{2} i \sin \varphi\left(\frac{n_{2} \cos \theta_{2}}{n_{1} \cos \theta_{1}}-\frac{n_{3} \cos \theta_{3}}{n_{2} \cos \theta_{2}}\right) \\
& M_{22}=\frac{1}{2}\left(1+\frac{n_{3} \cos \theta_{3}}{n_{1} \cos \theta_{1}}\right) \cos \varphi-\frac{1}{2} i \sin \varphi\left(\frac{n_{2} \cos \theta_{2}}{n_{1} \cos \theta_{1}}-\frac{n_{3} \cos \theta_{3}}{n_{2} \cos \theta_{2}}\right)
\end{aligned}
$$

The transmittance and reflectance are written as:

$$
\begin{aligned}
& T=\frac{1}{M_{11}} \\
& R=\frac{M_{21}}{M_{11}}
\end{aligned}
$$

\section{Single junction solar cells}

The authors utilize the AM1.5 as a standard condition for investigating the performance of solar cells. In AM1.5 angle of radiation of 48.2 and light intensity is around $100 \mathrm{~mW} / \mathrm{cm}^{2}$ [6]. The current-voltage (I-V) characteristics of $\mathrm{p}-\mathrm{n}$ junction solar cells under steady-state illumination can most simply be described using the single exponential model as,

$$
J=-J_{p h}+J_{0}\left(e^{q v / n K T}-1\right)
$$

Where $J_{p h}$ represents the photo-generated current density, $\mathrm{V}$ is the terminal voltage, i.e., the voltage developed across the junction, $\mathrm{k}$ is the Boltzmann constant and $\mathrm{n}$ is the ideality factor. The open-circuit voltage is the maximum voltage available from a solar cell. Equation (1) at $J=0$ yields the expression for $V_{\text {oc }}$ as:

$V_{o c}=\frac{K T}{q} \operatorname{In}\left(\frac{J_{s c}}{J_{0}}+1\right)$

Fill Factor is defined as the ratio of the maximum power output at the maximum power point to the product of the opencircuit voltage and short-circuit current density and can be expressed as:

$$
F F=\frac{P_{\max }}{V_{o c} J_{s c}}
$$

The efficiency of a solar cell is the ratio of the power output corresponding to the maximum power point to the power input and is represented as: 
$\eta=\frac{V_{o c} J_{s c} F F}{p_{i n}}$

Where $P_{\text {in }}$ is the intensity of the incident radiation [7]. So it results that we decrease the shinning and reflecting while promoting the power conversion efficiency of the solar cell. For decreasing reflection from the surface of the cell, two kinds of materials, $\mathrm{SiO}_{2}$ and $\mathrm{Si}_{3} \mathrm{~N}_{4}$ with a thickness of $d_{1}=0.1 \mu \mathrm{m}$ and $d_{2}=0.2 \mu \mathrm{m}$, and $n_{1}=1, n_{2}=1.45, n_{3}=1.92$ and $n_{4}=3.4$ is investigated. In this study, the focus is on using consumable and practically usable materials. The designed filter could be over $400 \mathrm{~nm}$ and enters a greater amount of light into the cell. The applied filter is shown in Fig. 2.

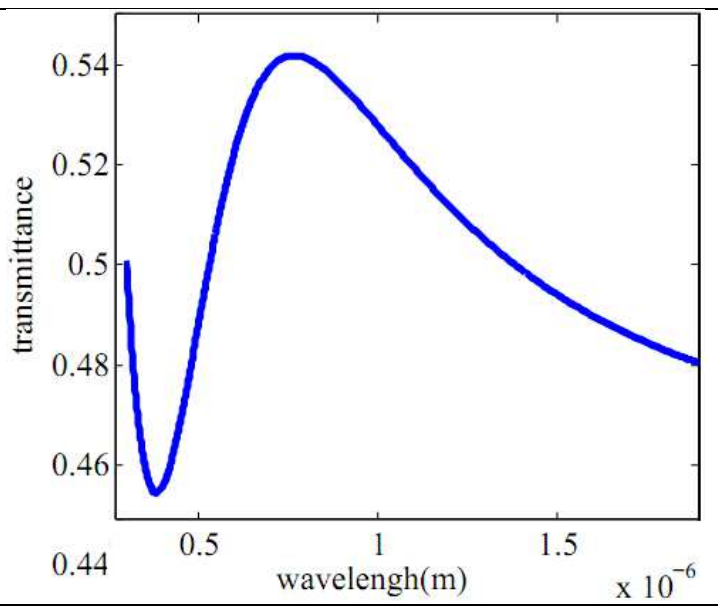

Fig. 2. The designed Chart filters using compounds of $\mathrm{SiO}_{2}$ and $\mathrm{Si}_{3} \mathrm{~N}_{4}$, with a thickness of $0.1 \mu \mathrm{m}$ and $0.2 \mu \mathrm{m}$.

With attention to this, the general material of the solar cells is silicon and it does not affect the doping level in the refractive index structure. Fig. $\mathbf{2}$ is showing the transmittance component of incident light of the designed filter versus wavelength. In the range between $400 \mathrm{~nm}$ to $800 \mathrm{~nm}$, the transmittance is increased and reached its maximum point. A magnificent point is that our designed filter well matches the spectrum of light. Fig. $\mathbf{3}$ shows the wavelength of the solar spectrum in the standard condition of AM1.5. 


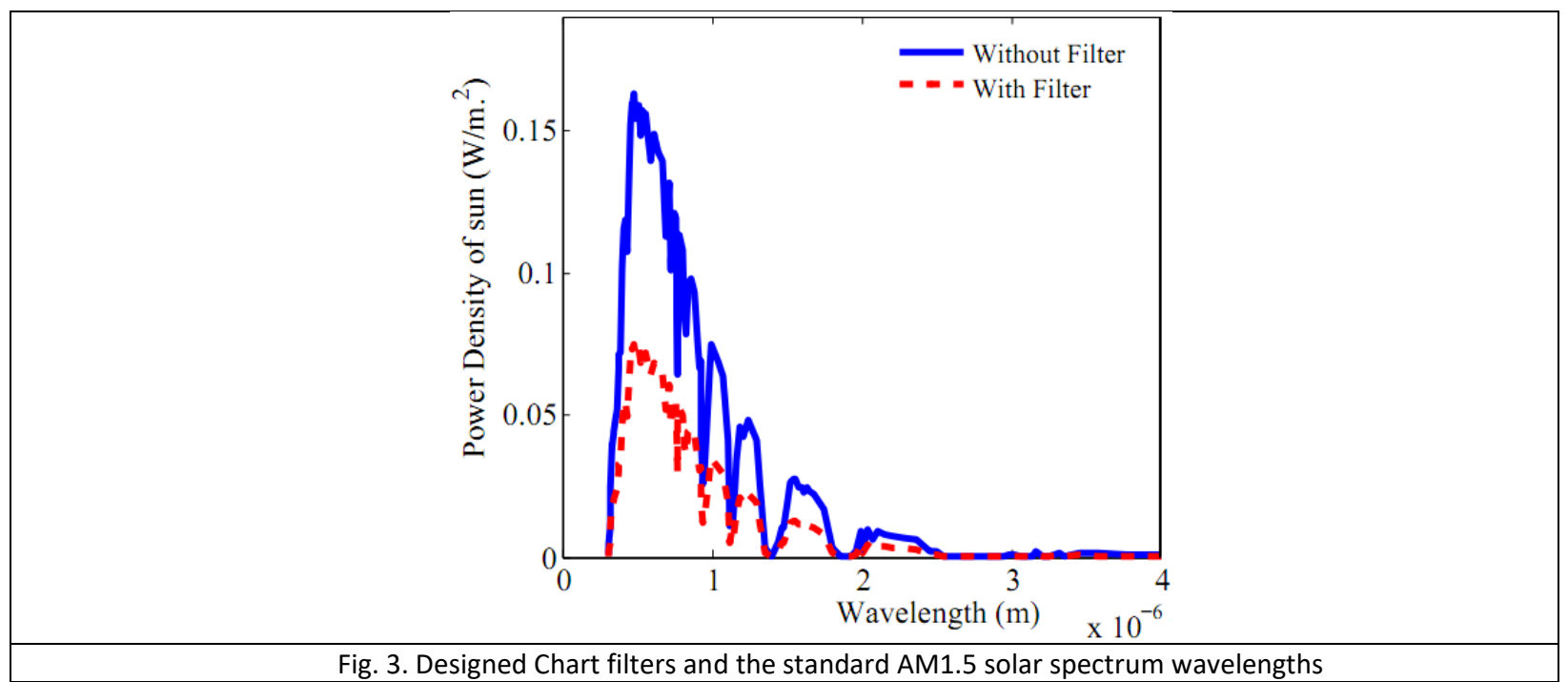

From the Varshney model the bandgap of semiconductors is changed with temperature variations [8]:

$\varepsilon_{C V}(T)=\varepsilon_{C V}(0)-\frac{\alpha T^{2}}{T+\beta}$

Where $\varepsilon_{\mathrm{cv}}(\mathrm{T})$ and $\varepsilon_{\mathrm{cv}}(0)$ are the band gaps at temperature T and $0 \mathrm{~K}$ respectively, $\alpha$ and $\beta$ [9] are constants and their values for Si and 3C-SiC are listed in Table 1 [10].

Table 1. Parameters of Si and 3C-SiC

\begin{tabular}{|c|c|c|c|}
\hline Material & $\varepsilon_{\mathrm{cv}}(0)(\mathrm{eV})$ & $\alpha\left(\mathrm{eVK}^{-1}\right) \times 10^{-4}$ & $\beta(\mathrm{K})$ \\
\hline $\mathrm{Si}$ & 1.16 & 7.02 & 1108 \\
\hline 3C-SiC & 2.2 & 6 & 1200 \\
\hline
\end{tabular}

To investigate the impact of temperature on solar cells, we assume the Si and SiC-based solar cells. The efficiency versus temperature is traced in Fig. $\mathbf{3}$ with and without using the filter. 


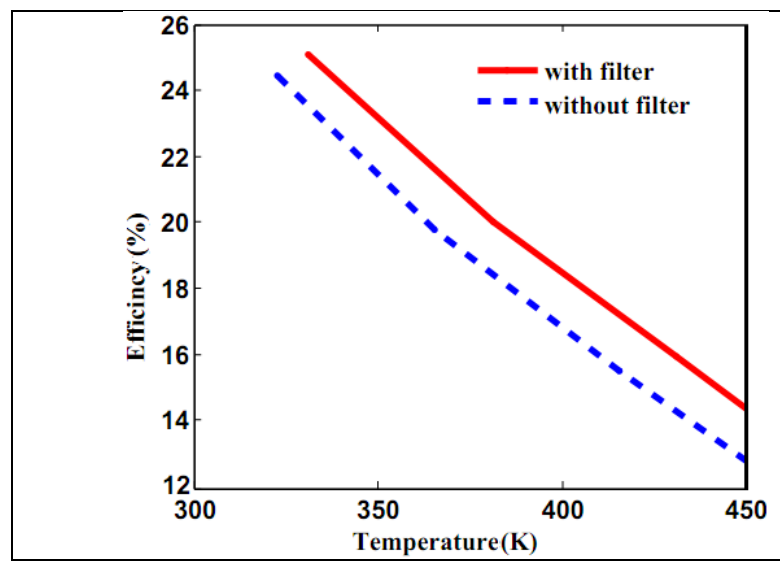

(a)



(b)

Fig. 3. Efficiency versus ambient temperature in both filtered and unfiltered (a) Si and (b) 3C-SiC based solar cells

As shown in figure 3 the efficiency with using the filter is higher than the contrast without using a filter and the power conversion efficiency is also improved. The $3 \mathrm{C}$-SiC solar cell-based system possesses some favorable characteristics that make it suitable and applicable substitute material in photovoltaic systems. In addition, the 3C$\mathrm{SiC}$ solar cell is potential material, for a high-temperature application, wider bandgap semiconductor i.e. $2.2 \mathrm{eV}$ for $3 \mathrm{C}-\mathrm{SiC}$ and $3 \mathrm{eV}$ for $6 \mathrm{H}-\mathrm{SiC}$, and comparable carrier mobility with Si [11].

\section{Temperature effect on Intermediate Band Solar Cells}

Intermediate Band Solar Cells (IBSCs) are another type of solar cells introduced by Luque in 1997 [12] harvest most parts of the electromagnetic spectrum by a mini sandwiched band between valance and conduction band. The schematic diagram of an IBSC is shown in Fig. 4 in which there are three transitions, (i) transition between valance band and intermediate band (ii) between intermediate band and conduction band (iii) between valance and conduction band.

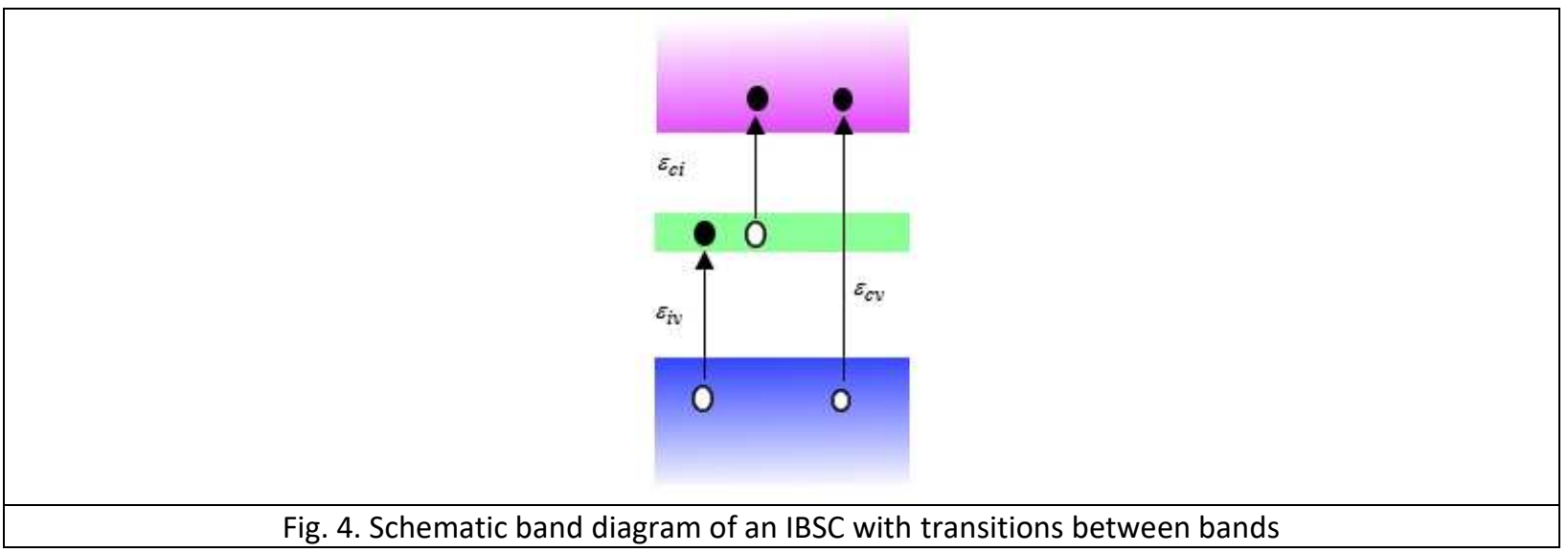


To analyze the cell performance and efficiency calculation the authors of this investigation use the Detailed Balance model introduced by Shockley and Queisser [13]. From the Detailed Balance model the output current is the difference between the absorbed and emitted current and can be written as:

$$
\begin{gathered}
J_{\text {out }}=J_{a b s}-J_{\text {em }} \\
J_{a b s}=q \int_{\varepsilon_{c v}}^{\infty} N\left(\varepsilon, 0, T_{s}\right) d \varepsilon \\
J_{e m}=q \int_{\varepsilon_{c v}}^{\infty} N\left(\varepsilon, \mu, T_{c}\right) d \varepsilon
\end{gathered}
$$

Where $N(\varepsilon, \mu, T)$ is the particle flow calculated from Planck's law [14]:

$$
N(\varepsilon, \mu, T)=\frac{2 \pi}{h^{3} C^{2}} \frac{\varepsilon^{2}}{\exp ((\varepsilon-\mu) / \mathrm{KT})-1}
$$

Where $C$ is the speed of light, $h$ is Planck's constant, $K$ is Boltzmann's constant, and $T_{s}$ and $T_{c}$ are the temperature of sun and cell respectively. $\mu$ is the chemical potential and in the case of solar radiation is zero but in luminescent emission from a photovoltaic device equals $\mathrm{eV}$, where $e$ is the charge of the electron and $V$ is the voltage across the device. Output Power is the product of $J$ with $V$ and power conversion efficiency is:

$$
\eta=p_{\text {out }} / \int_{0}^{\infty} \varepsilon N\left(\varepsilon, 0, T_{S}\right) d \varepsilon
$$

The efficiency against sub-bandgaps is traced in Fig. 5. The maximum efficiency is occurred in the $E_{c i}=0.71$ eV and $\mathrm{E}_{\mathrm{iv}}=1.24 \mathrm{eV}$ which is about \%63.

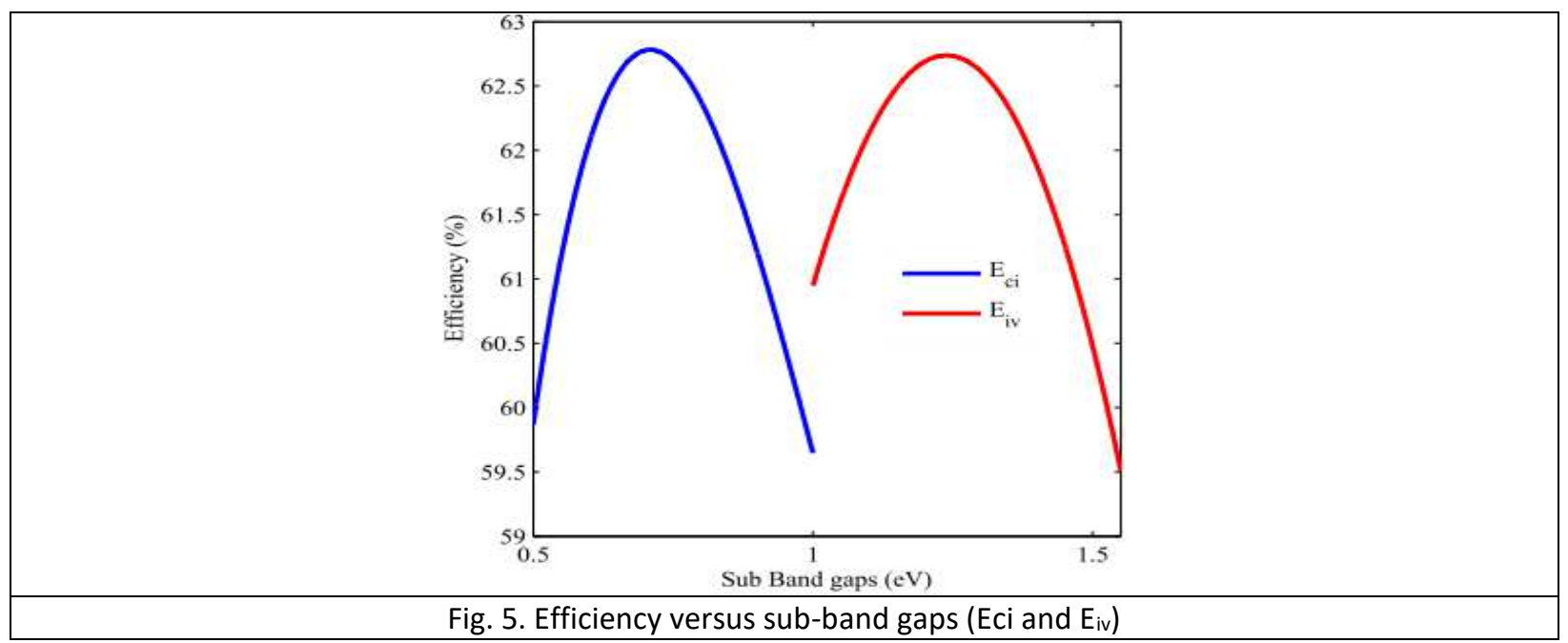


To verify the impact of temperature on IBSC we consider two sources of heat, (i) external induced temperature due to direct radiation of the sun and (ii) internally generated temperature due to high energy photons which produce hot carriers, and if they aren't extracted rapidly they thermalize the lattice and destruct the cell performance.

\subsection{External temperature effect}

Similar to single-junction solar cells the IBSCs are suffering from temperature increment. Throughout using the Varshni model we can verify the temperature effect on IBSCs. As stated previously the bandgap of semiconductors is decreased by temperature increment. Contour plot of efficiency variations versus two sub-band gaps ( $E_{c i}$ and $\left.E_{i v}\right)$ is shown in Fig. 6. As shown in Fig. 6 (a) at $300 \mathrm{~K}$ the $3 \mathrm{C}-\mathrm{SiC}$ curve is traced in a dashed line. If $\mathrm{E}_{\mathrm{iv}}$ and $\mathrm{E}_{\mathrm{ci}}$ are $0.8 \mathrm{eV}$ and $1.35 \mathrm{eV}$ the efficiency is about $62 \%$. While, at $800 \mathrm{~K} \mathrm{E}_{\mathrm{iv}}$ and $\mathrm{E}_{\mathrm{ci}}$ are $0.75 \mathrm{eV}$ and $1.25 \mathrm{eV}$, respectively, and the efficiency will reduce to about $49 \%$.

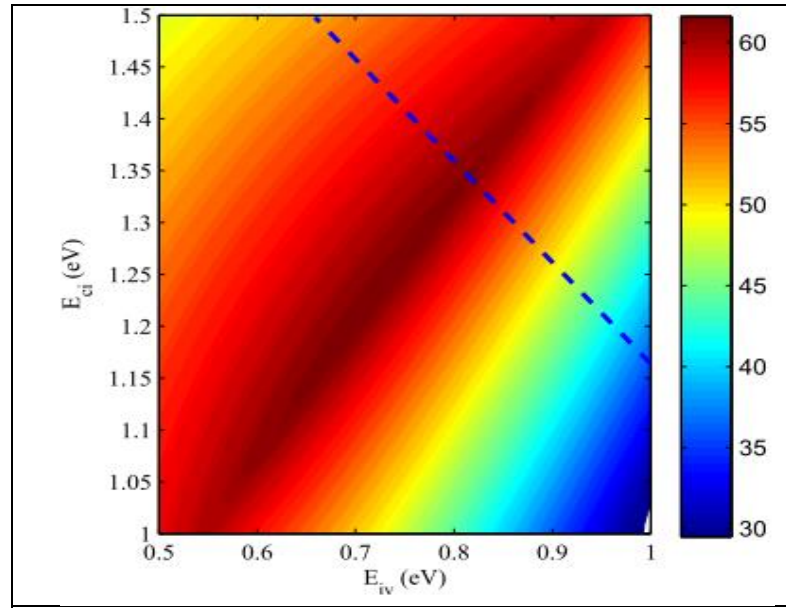

(a)

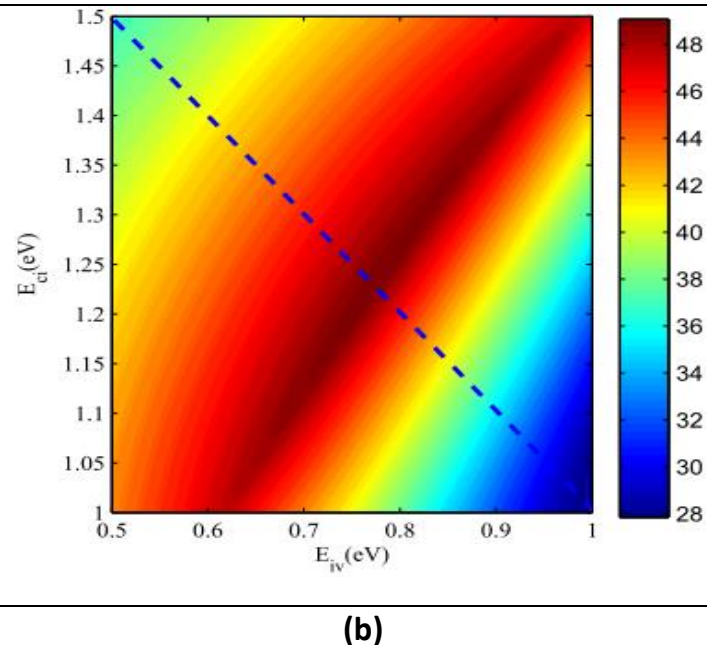

(b)

Fig. 6. Contour plot of an IBSC versus sub-band gaps dashed lines are showing bandgap of 3C-SiC at (a) $300 \mathrm{~K}$ and (b) $800 \mathrm{~K}$.

To overcome the impact of temperature on the solar cell the authors utilized the filter on the solar cell. The efficiency against temperature for an IBSC based on $3 \mathrm{C}-\mathrm{SiC}$ is traced in Fig. 7 with filter and without it. It results that by using filter the efficiency stability is higher and its decrement is slower than without using it. 


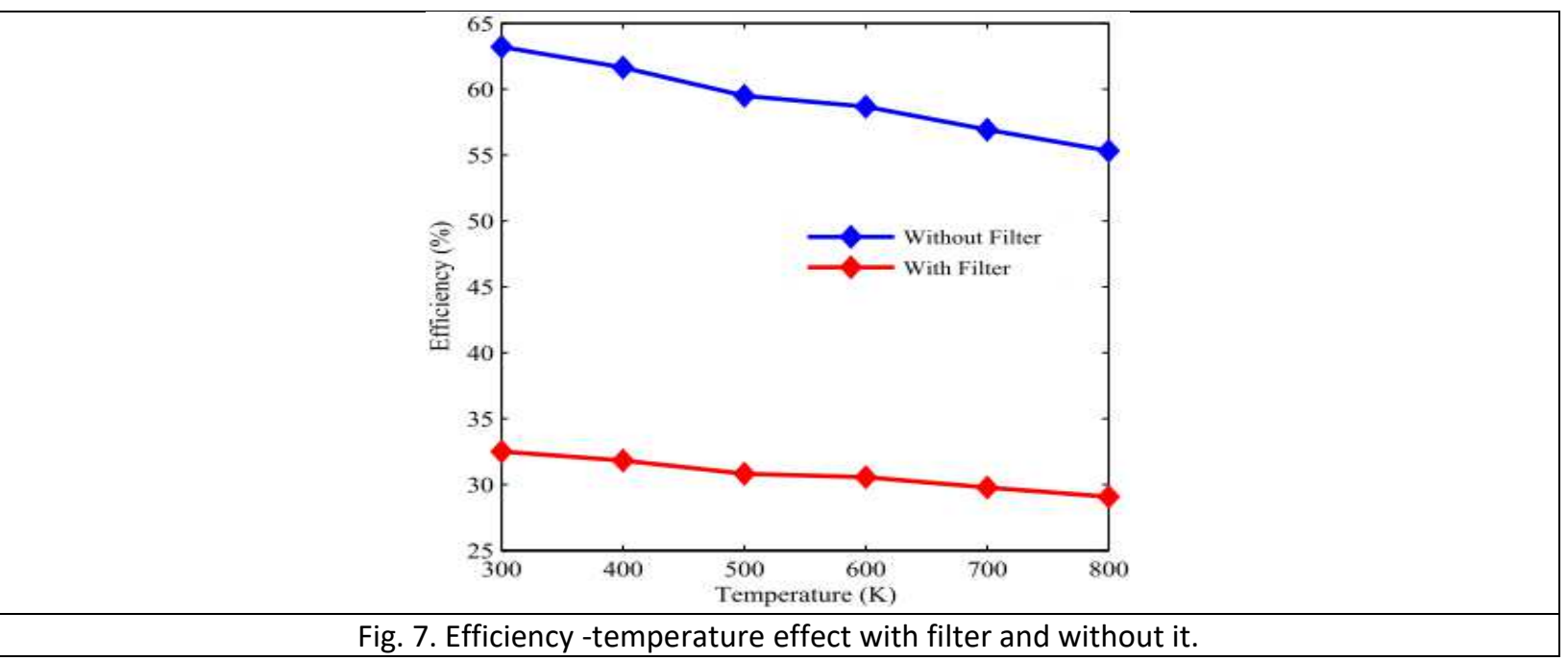

\subsection{Internal temperature effect}

The internal temperature can be generated through high-energy photons which create hot carriers. Extracting of the hot carrier must be extremely fast, to prevent the thermalization of the carrier [15]. For this case, we use energy selective contacts (ESCs) to extract more and more hot carriers. ESCs are double barrier tunneling junctions that are made with $\mathrm{SiO}_{2}$ sandwiched between $\mathrm{Si}[16,17]$. The schematic band diagram of the hot carrier extracting is shown in Fig. 8. Here, we assume multilevel implanted ESCs on the valance and conduction band. We use a novel method for extracting the high-energy carrier by using ESCs implanted with specific intervals and supposing the width of each ESC is small.

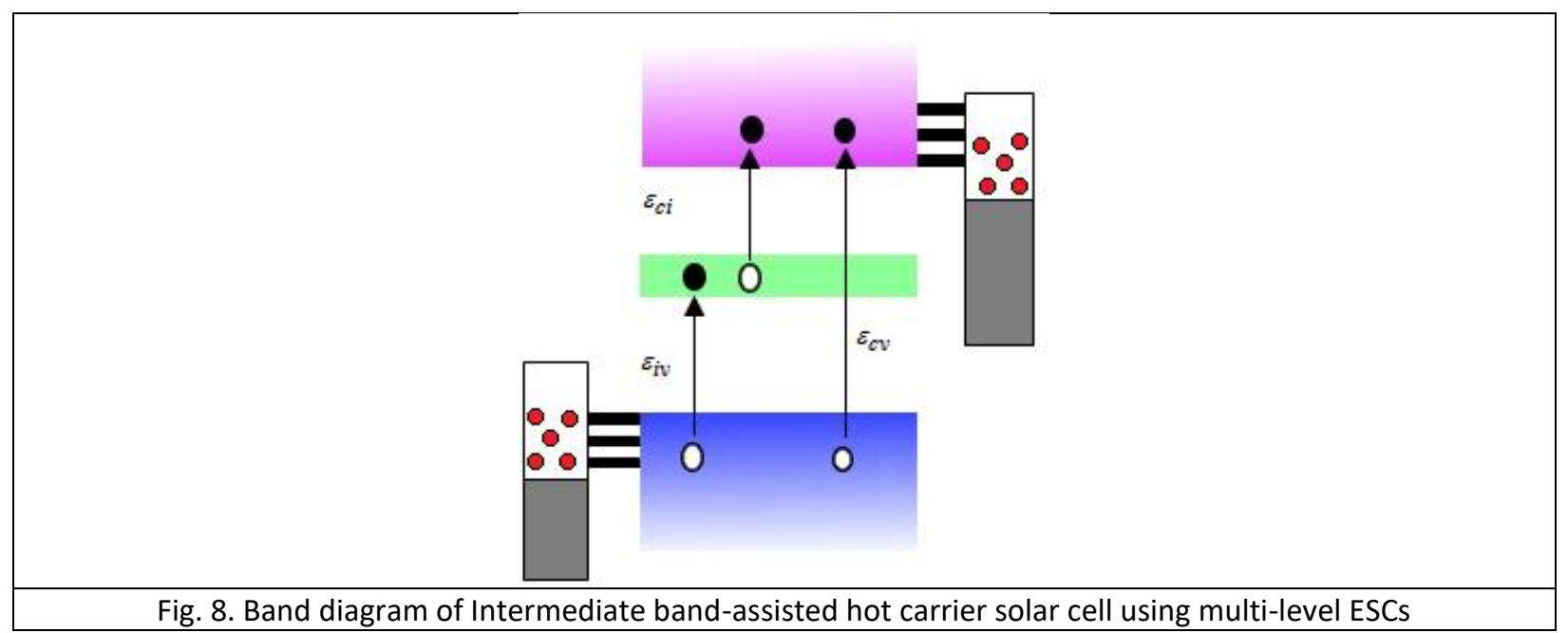

Using selective energy contact, the generated heat inside the solar cell is decreased and the following relation (Eq. 22) shows the amount of the heat reduction. In this equation $\Delta E_{i}$ is the energy difference between contact $i+1$ and I and also $\lambda_{i}$ is wavelength corresponds to contact $i+1$. 
$\Delta E_{\text {Thermal }}=\Delta E_{1} \int_{\lambda_{1}}^{\lambda_{g}} D(E) \cdot F(E) d E+\Delta E_{2} \int_{\lambda_{2}}^{\lambda_{1}} D(E) \cdot F(E) d E+\ldots \ldots$

The effect of several ESCs is lowering the thermalization due to extracting of hot carrier rapidly. The efficiency of using several ESCs in different ESCs is shown in Fig. 9.

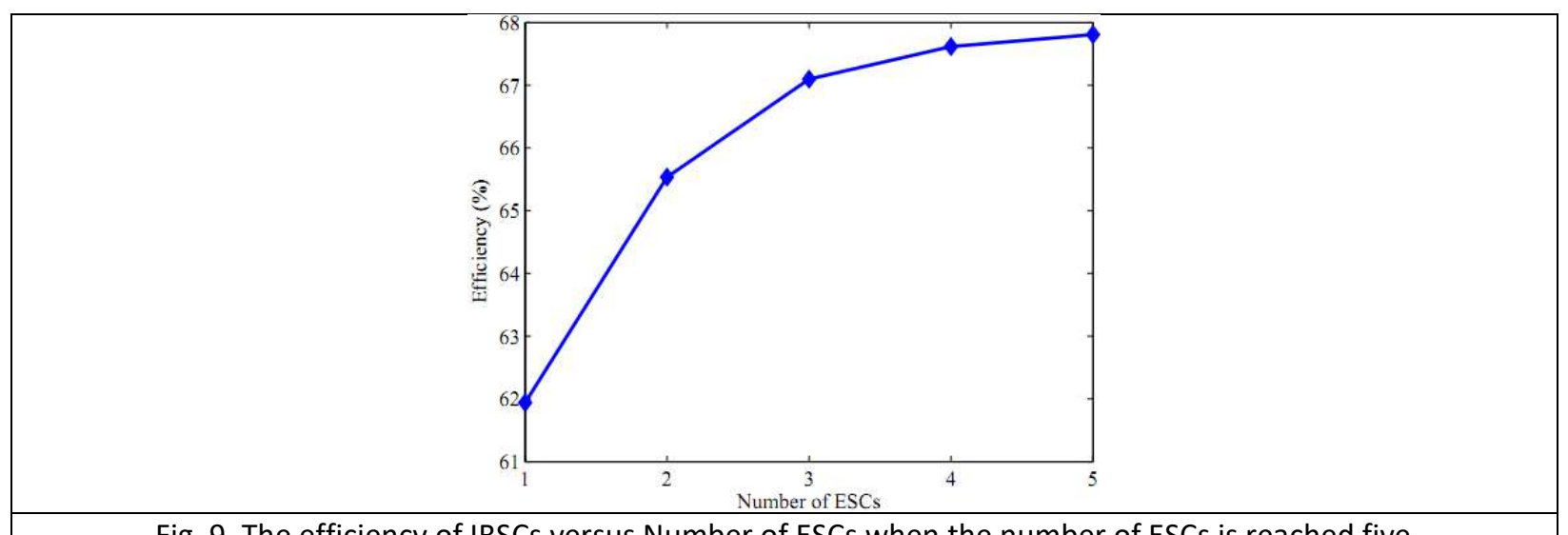

Fig. 9. The efficiency of IBSCs versus Number of ESCs when the number of ESCs is reached five

Fig. 9 shows the efficiency contrasted with the number of ESCs using multilevel ESCs. The Efficiency is increased due to the extraction of the hot carrier from different energy levels in conduction and valance band. The extracted efficiency without using any ESCs is about \%62 while by using five ESCs it is increased to \%68.

\section{Conclusion}

This study investigated the efficiency of solar cells with and without optical filters (multilayer stack) in front of the cell. We used the optical filter on the top surface of Si and SiC solar cells. The results show that the filter has a significant effect on the efficiency enhancement. The efficiency variations with temperature were investigated and we observed that the SiC solar cells are better than Si due to their stability in $450 \mathrm{~K}$. Similarly, another type of solar cell, namely IBSCs, was investigated, and the designed filter was applied to it. As a result, the efficiency was increased dramatically and it becomes more stable at the same time. The other source of energy is the internal energy, which can be created due to hot carriers, which was decreased by using the multilevel ESCs. Finally, the multilevel ESCs are lowering the thermalization of the carriers, increasing the efficiency and breakdowns the Shockley-Queisser limit.

\section{References}

[1] Wei Zhou, Hongxing Yang, Zhaohong Fang, A novel model for photovoltaic ARRAY PERFORMANCE prediction, Received 26 October 2006; received in revised form 20 April 2007; accepted 21 April 2007.

[2] Foster R, Ghassemi M, Cota A. Solar energy-renewable energy and the environment, Taylor and Francis Group, 2010.

[3] Skoplaki E, Palyvos J A., On the temperature dependence of photovoltaic module electrical performance: a review of efficiency, power correlations. Solar Energy Materials \& Solar cells 2009; 83:614-24.

[4] Pochi Yeh, 2005, Optical wave in layered Media, A Wiley-Inter science Publication, 0471731927 
[5] Van Dyk EE et al. Temperature-dependent performance of crystalline silicon photovoltaic modules. S Afr J Sci 2000; 96: 198200.

[6] Antonio Luque and Steven Hegedus, 2003, Handbook of Photovoltaic Science and Engineering, John Wiley and Sons.

[7] Priyanka Singh, N. M. Ravindra, 2012, the Temperature dependence of solar cell performance an analysis, Solar Energy Materials \& Solar Cells 101 (2012) 36-45

[8] Katherin leung ray, photovoltaic cell efficiency at elevated temperature, Massachusetts institute of technology June2010.

[9] B. Pejova, B. Abay, and I. Bineva, Temperature Dependence of the Band-Gap Energy and Sub-Band-Gap Absorption Tails in Strongly Quantized ZnSe Nanocrystals Deposited as Thin Films, J. Phys. Chem. C114(36), 15280-15289 (2010). 3.

[10] M. Bhatnagar, B. J, Baliga, Comparison of $6 \mathrm{H}-\mathrm{SiC}, 3 \mathrm{C}-\mathrm{SiC}$, and Si for power devices. IEEE Transaction on Electron Devices 40(1993) 645-655

[11] A. El-Shaer, M. T. Y. Tadros, M. A. Khalifa ,Effect of Light intensity and Temperature on Crystalline Silicon Solar Modules Parameters, International Journal of Emerging Technology and Advanced Engineering Website: www.ijetae.com (ISSN 22502459, ISO 9001:2008 Certified Journal, Volume 4, Issue 8, August 2014)

[12] Luque, A. \& Marti, A., 1997. Increasing the efficiency of ideal solar cells by photon-induced transitions at intermediate levels. Physical Review Letters, 78(26), 5014.

[13] Shockley, W., Queisser, H. J., 1961. Detailed balance limit of efficiency of p-n junction solar cells. Journal of applied physics, 32(3), 510-519.

[14] Green, M. A., 2006. Third-generation photovoltaic: advanced solar energy conversion (Vol. 12). Springer.

[15] Ross, R. T., Nozik, A. J., 1982. The efficiency of hot carrier solar energy converters. Journal of Applied Physics, 53(5), 38133818.

[16] Shrestha, S. K., Aliberti, P., and Conibeer, G. J., 2010. Energy selective contacts for hot carrier solar cells. Solar Energy Materials and Solar Cells, 94(9), 1546-1550.

[17] Conibeer, G. J., Jiang, C. W., König, D., Shrestha, S., Walsh, T., \& Green, M. A., 2008. Selective energy contacts for hot carrier solar cells. Thin Solid Films, 516(20), 6968-6973. 